\title{
Efficacy, patients' perception, and cost of medication in allergic rhinitis with subcutaneous immunotherapy
}

\author{
Karnsinee Thanborisutkul, ${ }^{1}$ Nantika Khodtecha, ${ }^{2}$ Prapasri Kulalert, ${ }^{3}$ Paskorn Sritipsukho ${ }^{4,5}$ \\ Orapan Poachanukoon, ${ }^{2,5}$ Sira Nanthapisal ${ }^{5}$
}

\begin{abstract}
Background: Allergic rhinitis (AR) is the allergic inflammation of nasal mucosa. Treatment of AR includes pharmacotherapy and allergen immunotherapy. Subcutaneous immunotherapy (SCIT) is indicated in inadequate disease control, patient's preference, or impossible allergen avoidance. SCIT is an effective treatment but its cost is comparatively high. Efficacy, patient perception, and cost of medication are rarely explored in Asia.
\end{abstract}

Objectives: To study efficacy, patient perception, and cost-benefit of SCIT in AR

Methods: We performed a descriptive cross-sectional study at Thammasat University Hospital, Thailand. AR patients who had been receiving SCIT were interview. Current and recall of AR total symptom score (TSS), quality of life, and perception were scored. Cost of medications before SCIT and current cost were reviewed from the medical records.

Results: A total of 142 patients were enrolled. Sixty-eight patients (47.9\%) received single allergen; house dust mite was the most common allergen. The median of maintenance phase was 47 months, range 15-142 months. The mean of current TSS was significantly lower than mean TSS before SCIT. Forty-two patients (29.6\%) had discontinued SCIT on the day of the interview. After discontinuation of SCIT, TSS was still lower than TSS before SCIT. The average cost of medications including SCIT was lower than that of before SCIT with an average difference of 254.2 USD/year. Sixteen patients (11.3\%) experienced systemic reaction, 8 of which had reaction during rush immunotherapy.

Conclusions: SCIT is an effective, cost-saving and safe treatment option for AR. Rush immunotherapy can reduce duration of build-up phase but increase the risk of systemic reaction.

Key words: Immunotherapy, efficacy, cost-benefit, perception, safety

\section{From:}

Department of Pediatrics, Faculty of Medicine, Thammasat University, Pathumthani, Thailand

2 Thammasat university center of allergy, asthma, and pulmonary disease, Thammasat University Hospital, Pathumthani, Thailand

Department of clinical epidemiology, Faculty of Medicine,

Thammasat University, Pathumthani, Thailand

${ }^{4}$ Center of Excellence in Applied Epidemiology, Thammasat University, Thailand

Division of allergy, immunology and rheumatology,

Department of Pediatrics, Faculty of Medicine, Thammasat University

\section{Corresponding author:}

Sira Nanthapisal

99/206 Department of Pediatrics, Faculty of Medicine,

Thammasat University, Pathumthani, 12120 THAILAND

E-mail: nsira@tu.ac.th

\section{Introduction}

Allergic rhinitis (AR) is the inflammation of nasal mucosa induced by hypersensitivity to inhalant allergens and one of the most common respiratory problems worldwide and Thailand. ${ }^{1,2}$ Cardinal nasal symptoms of AR are itching, sneezing, nasal obstruction and nasal discharge. More than a half of AR individuals also complain of eye symptoms including eye irritation, redness and watery eyes. ${ }^{1,3}$ AR symptoms affect quality of life, sleep and performance of patients, social life, sleep, and performance at school/work. ${ }^{1,4}$ Diagnosis of AR is mainly based on clinical symptoms and presentations. Severity of AR is classified by: 1) duration of symptoms (persistent or intermittent) and 2) how AR affect quality of life (mild and moderate to severe). ${ }^{1}$ Causative allergens of AR are classified by the present of allergens into 2 groups: 1) indoor allergens e.g. 
house dust mite, cockroach, cat hair, dog epithelium, and mold 2) outdoor allergen e.g. pollen, grass, and mold. Individuals sensitsation can be evaluated by either prick skin test or serum specific IgE to the allergens. ${ }^{5,6}$ Treatment of allergic rhinitis should be a combination of pharmacotherapy, patient education, allergen avoidance and immunotherapy. ${ }^{1,6}$

Specific allergen immunotherapy (SIT) is a unique therapy for allergic rhinitis as it provides symptomatic relief and modify allergic inflammation by targeting the underlying immunologic mechanisms. ${ }^{7-9}$ Sublingual (SLIT) and subcutaneous (SCIT) immunotherapy are the two most commonly prescribed routes for administering SIT. ${ }^{9}$ The mechanisms of action of allergen-specific immunotherapy include desensitization effects, modulation of T-and B-cell responses and related antibody isotypes, and migration of eosinophil, basophils, and mast cells to tissues, as well as release of their mediators. ${ }^{8,10,11}$ Indications for SIT in the treatment of AR include 1) inadequate control of symptoms despite of appropriate pharmacotherapy and allergen avoidance, 2) patient's preference to reduce morbidity from $A R, 3)$ undesirable adverse effects from pharmacotherapy, and 4) complete allergen avoidance is impossible. ${ }^{12}$ SCIT administration are divided into 2 phases: 1) initial built-up phase: when the dose and concentration of allergen are increased and 2) maintenance phase: when patient receives effective therapeutic dose for a period of time. ${ }^{13}$ Benefits from SIT in AR include decreased both nasal and eye symptoms, improved quality of life, decreased medication used. Furthermore, SIT may prevent the development of allergic asthma in patient with $\mathrm{AR}$ and reduce development of new sensitisations. ${ }^{8,12,13}$ Adverse effects of SCIT range from local reaction which are swelling and itching around injected site to systemic reaction like bronchospasm, angioedema or anaphylaxis. ${ }^{13}$ Only few studies demonstrate patients' aspect on satisfaction, knowledge, and quality of life after the maintenance phase. In Thailand, there are very few studies on the cost-benefit of SCIT in AR individuals.

The aim of this study is to assess the improvement of AR symptoms during maintenance phase of SCIT, quality of life, satisfaction and cost of AR treatment for to improve the quality of patient care in the future.

\section{Methods}

A descriptive cross-sectional study was conducted from March to August, 2019 at Thammasat University hospital, Pathumthani, Thailand. The study was approved by Institutional ethical committee MTU-EC-PE-0-169/61. Inform consent was obtained before enrollment. Patients with AR diagnosed by clinical presentations and allergen sensitisation based on positive skin prick test (a positive result defined as a wheal $\geq$ $3 \mathrm{~mm}$ diameter) or serum specific immunoglobulin $\mathrm{E}$ (IgE) (a positive result defined as $\mathrm{IgE}$ level $\geq 3.5 \mathrm{kUA} / \mathrm{L}$ ) were interviewed either by phone or personal interview. All patients were sensitised to at least one aeroallergen. Patients had been treated with SCIT and, by the time of interview, were either on the maintenance phase or discontinued the maintenance phase. Medical records of patients were reviewed. Patients demographics and clinical data were recorded. Medicine prescription, and adverse effect of SCIT were collected from medical records. Cost of medications and SCIT were reviewed and calculated from medical record, doctor prescription and hospital drug price list. Recall of before-SCIT and current total nasal symptom score (TNSS), total ocular symptom score (TOSS), total symptoms score (TSS; equal to TNSS + TOSS), questionnaire for quality of life of allergic rhinitis, perception and satisfaction on SCIT were scored on the day of interview. Recall symptom scores before SCIT were also compared with the medical records prior to SCIT to reassure the score was correlated with the severity of disease before SCIT. Patients with incomplete data were excluded from the study. Symptom score items, questionnaire for quality of life of allergic rhinitis, questions for perception and satisfaction on SCIT are presented in supplementary data.

Category data were compared using Fisher's exact or Chi-square test when appropriate. Continuous variables were compared by T-Test. A 2 -sided $\mathrm{p}$ value $<0.05$ was considered statistically significant.

\section{Results \\ Demographic data}

A total of 142 patients were enrolled in the study. Female to male ratio was $1: 1.95$. Mean age was $18.28( \pm 10.73, \mathrm{SD})$ years. Severity of AR before SCIT according to ARIA classification were mild intermittent in 8 patients $(5.6 \%)$, mild persistent in 18 patients (12.6\%), moderate to severe intermittent in 77 patients $(54.2 \%)$, and moderate to severe persistent in 39 patients (27.5\%). Median duration of AR was 37.04 (8.4649.8, IQR) months. Other allergic diseases were identified as follow: asthma in 43 patients (30.3\%); atopic dermatitis in 22 patients (15.5\%); food allergy in 8 patients $(5.6 \%)$. The most common sensitized allergen was house dust mite (Dermatophagoides pteronyssinus $90.1 \%$ and Dermatophagoides farinae $85.2 \%$ ), following by cockroach (American cockroach $44.4 \%$ and German cockroach $38.7 \%$ ), grass $22.5 \%$, cat hair $6.3 \%$, dog epithelium $2.8 \%$, and others $1.4 \%$. (Table 1 )

\section{Table 1. Demographics and clinical data}

\begin{tabular}{lc}
\multicolumn{1}{c}{ Characteristics } & $\begin{array}{c}\text { N }=142, \\
\text { Number (\%) }\end{array}$ \\
\hline Gender & $94(66.2)$ \\
- Male & $48(33.8)$ \\
- Female & $18.28 \pm 10.73$ \\
Mean age \pm SD (years) & $47.20 \pm 23.06$ \\
Mean duration of treatment \pm SD (months) & \\
\hline Comorbid allergic disease & $43(30.3)$ \\
- Asthma & $22(15.5)$ \\
- Atopic dermatitis & $8(5.6)$ \\
\hline - Food/Drug allergy & \\
\hline AR severity before SCIT & $8(5.6)$ \\
\hline
\end{tabular}


Table 1. (Continued)

\begin{tabular}{|c|c|}
\hline Characteristics & $\begin{array}{c}\mathrm{N}=142, \\
\text { Number }(\%)\end{array}$ \\
\hline \multicolumn{2}{|l|}{ AR severity before SCIT (Continued) } \\
\hline - Mild persistent & $77(54.2)$ \\
\hline - Moderate to severe persistent & $39(27.5)$ \\
\hline \multicolumn{2}{|l|}{ Skin prick test } \\
\hline - Dermatophagoides pteronyssinnus & $128(92.9)$ \\
\hline - Dermatophagoides farinae & $121(89.4)$ \\
\hline - American cockroach & $63(44.7)$ \\
\hline - German cockroach & $55(41.2)$ \\
\hline - Bermuda & $15(10.6)$ \\
\hline - Timothy grass & $9(5.9)$ \\
\hline - Cat hair & $9(5.9)$ \\
\hline - Dog epithelium & $4(1.2)$ \\
\hline - Acacia & $4(1.2)$ \\
\hline - Careless weed & $4(1.2)$ \\
\hline - Kapok & $1(1.2)$ \\
\hline - Mold mix & $1(1.2)$ \\
\hline \multicolumn{2}{|l|}{ Medication before SCIT } \\
\hline Monotherapy & 0 \\
\hline \multicolumn{2}{|l|}{ Combination } \\
\hline - Antihistamine + Montelukast & $3(2.1)$ \\
\hline - Antihistamine + Intranasal steroid & $52(36.1)$ \\
\hline - Antihistamine + Intranasal steroid + Montelukast & $87(61.3)$ \\
\hline \multicolumn{2}{|l|}{ Type of allergen for SCIT } \\
\hline Single allergen & $68(47.9)$ \\
\hline - Mixed house dust mite & $56(39.4)$ \\
\hline - Mixed cockroach & $7(4.9)$ \\
\hline - Mixed grass & $5(4.2)$ \\
\hline Multiple allergen & $74(52.1)$ \\
\hline - Mixed house dust mite + Mixed cockroach & $61(43)$ \\
\hline - Mixed house dust mite + Mixed grass & $4(2.8)$ \\
\hline $\begin{array}{l}\text { - Mixed house dust mite }+ \text { Mixed cockroach }+ \\
\text { Mixed grass }\end{array}$ & $9(6.3)$ \\
\hline
\end{tabular}

Abbreviations: AR: allergic rhinitis, SCIT: subcutaneous immunotherapy

All patients had used combined medication before receiving SCIT: oral antihistamine $(\mathrm{OAH})$ and intranasal steroid (INS) in 52 patients (36.1\%); OAH and montelukast in 3 patients $(2.1 \%)$; and $\mathrm{OAH}+\mathrm{INS}+$ montelukast in 87 patients (61.3\%). (Table 1)

\section{Immunotherapy administration and allergens}

Frequency of immunotherapy administration during buildup phase was: conventional administration schedule (once a week) in 125 patients (88.74\%) and rush immunotherapy (administration of all build-up phase in 1week admission) in 16 patients (11.26\%). Sixty-eight patients (48.9\%) received single allergen: mixed HDM in 56 patients, cockroaches in 7 patients, and mixed grass in 5 patients. Seventy-four patients (51.1\%) received multiple allergens: mixed HDM \& mixed cockroaches in 61 patients; HDM \& mixed grass in 4 patients; and HDM \& mixed cockroaches \& mixed grass in 9 patients (details of allergen, concentration, dose and schedule of each build-up phase were described in supplementary data) Average duration of maintenance phase was 47.20 months $( \pm 23.06$; SD). Forty-two patients $(29.6 \%)$ had discontinued the maintenance phase SCIT on the day of interview; median of duration of maintenance phase in these patients was 47 months (range 15-142).

\section{Symptom score and quality of life}

Average TNSS (mean \pm SEM) decreased significantly after receiving SCIT (figure 1A), pre SCIT at $7.83 \pm 0.18$ vs post SCIT at $3.86 \pm 0.16(p<0.0001,95 \%$ CI 3.660 to 4.283$)$. Average TOSS (mean \pm SEM) before SCIT also decreased significantly compared to current TOSS (Figure 1B), $3.87 \pm 0.18$ vs $1.59 \pm 1.52(p<0.0001,95 \%$ CI 2.027 to 2.537$)$. With similar results, average TSS (mean \pm SEM) before SCIT was significantly decreased after receiving SCIT (Figure 1C), pre SCIT at 11.7 \pm 12.28 vs post SCIT at $5.44 \pm 0.24(p<0.0001,95 \%$ CI 5.843 to 6.664$)$.

In 100 patients $(70.4 \%)$ who were still receiving maintenance phase, average TSS on the day of interview were significantly lower than TSS prior to SCIT. TSS were significantly lower than TSS prior to SCIT since the first year of maintenance phase. There was also no difference of TSS at year 1 up to $>4$ years of maintenance phase (Figure $\mathbf{S} \mathbf{1}$ in supplementary data). There was no difference of TSS between single allergen SCIT and multiple allergen SCIT and among administered allergens.

In the patients who discontinued SCIT $(n=42)$, average TSS (mean \pm SEM) scored on the day of interview was significantly lower than TSS prior to SCIT, $12.93 \pm 0.51$ and $6.37 \pm$ 0.43 , respectively ( $p<0.0001,95 \%$ CI -7.889 to -5.227 ). TSS (mean \pm SEM) scored on the day of interview in those who were still receiving maintenance SCIT was also significantly lower than TSS prior to SCIT $11.16 \pm 0.35$ and $5.04 \pm 0.27$, respectively $(p<0.0001,95 \% \mathrm{CI}-6.993$ to -5.250$)$. TSS scored on the day of interview in the discontinued group was slightly higher than those who were receiving maintenance SCIT, $p=0.011,95 \% \mathrm{CI}-2.350$ to -0.3136 (Figure 2A). TSS after discontinuation of SCIT was significantly lower than TSS prior to SCIT at 3-6 months, 6-12 months, 12-18 months, and > 18 month (Figure 2B). There was no difference of TSS in the patient who discontinued SCIT at 4 different time points, $p$ $=0.1208$. No difference of TSS after discontinuation of SCIT between single allergen SCIT and multiple allergen SCIT was found (data was not shown). 
A

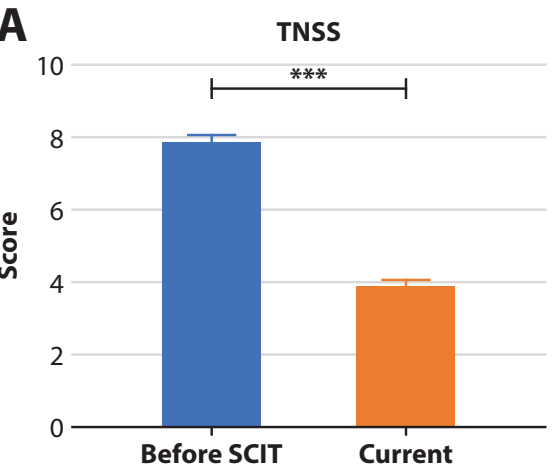

B

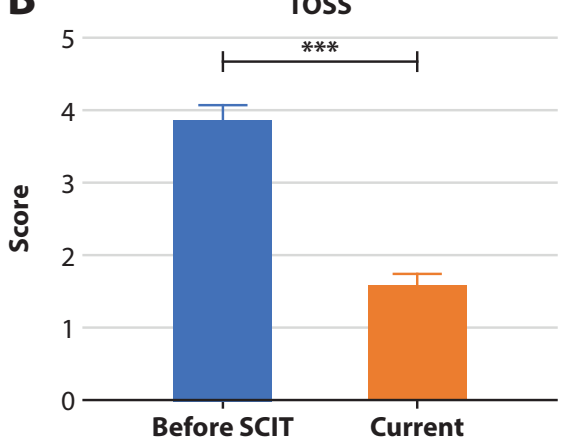

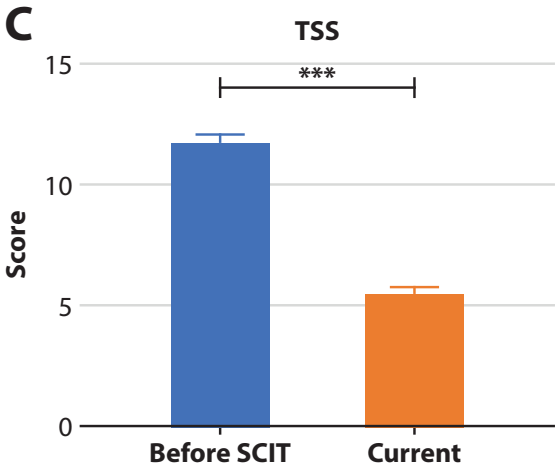

Figure 1. Symptom score before and after immunotherapy

A: current total nasal symptom score (TNSS) after subcutaneous immunotherapy (SCIT) was significantly lower than score before SCIT. B: current total ocular symptom score (TOSS) after SCIT was significantly lower than score before SCIT. C: current total symptom score (TSS) after SCIT was significantly lower than score before SCIT. ${ }^{\star \star \star} p<0.0001$
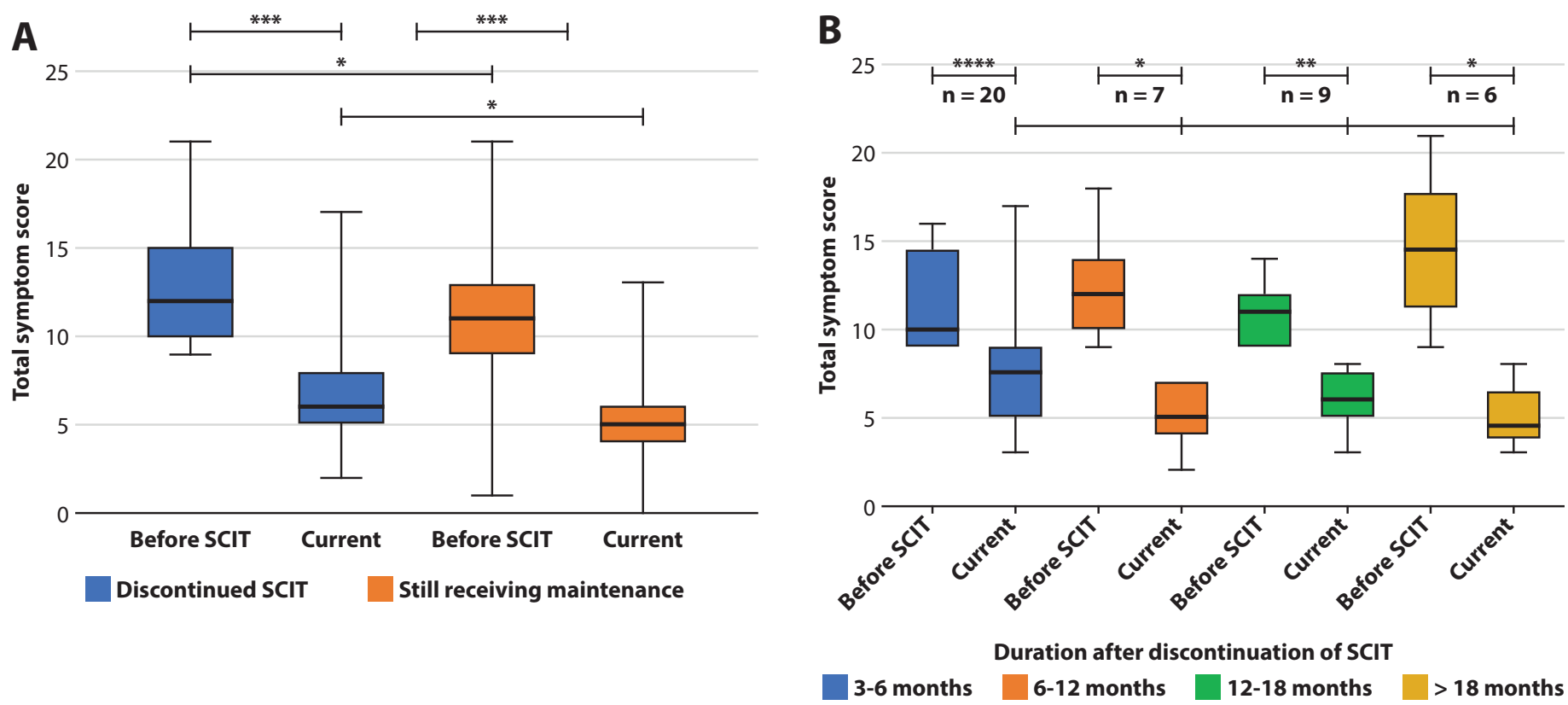

Figure 2. Comparison of total symptom score (TSS) before and after subcutaneous immunotherapy (SCIT)

A: Current TSS after the treatment with subcutaneous immunotherapy (SCIT) was significantly lower than the recall TSS before SCIT treatment in both patients who had discontinued SCIT and who were still receiving maintenance SCIT. Patients who were still receiving maintenance SCIT (pink) had lower current (TSS) than the patients who had discontinued SCIT (blue). B: TSS after discontinuation of SCIT was significantly lower than the recall TSS prior to SCIT in the patients who had discontinued SCIT for 3-6 months (blue), 6-12 months (pink), 12-18 months (green), and $>18$ months (yellow). There was no difference of TSS among the patients who had discontinued SCIT at 4 different time points. $\left({ }^{\star} p<0.05,{ }^{* *} p<0.01\right.$ and $\left.{ }^{* * * *} p<0.0001\right)$

Quality of life (QOL) assessed by Questionnaire for quality of life of allergic rhinitis was significantly improved; average QOL score had decreased from $29.74( \pm 0.90$, SEM) to 12.70 ( $\pm 0.70, \mathrm{SEM}$ ), $p<0.0001,95 \% \mathrm{CI} 15.84$ to 18.23 (Figure $\mathbf{S 2}$ in supplementary data).

\section{Cost of treatment and patient perspective on immunotherapy}

Average cost of medication for allergic rhinitis per year prior to SCIT which was 489.6 US dollar (USD) $( \pm 243.3$, SD) was significantly higher than average current cost of medication (including cost of immunotherapy) that was 235.4 USD ( $\pm 198.4, S D), p<0.0001,95 \%$ CI -303.7 to -204.7 (Figure 3A). Sixty-eight patients (47.9\%) were not prescribed any medicine at least 3 months prior to the interview, 24 of which discontinued SCIT. Difference of cost between before SCIT and current cost per year (including cost of immunotherapy) were plotted against difference of TSS before SCIT and current cost (Figure 3B); cost of treatment decreased $254.2 \mathrm{USD} /$ year by average and TSS also decreased 6.22 point.

Average VAS score of AR clinical improvement after receiving SCIT was $7.99( \pm 1.35$, SD). Patients were satisfied with overall adverse effects of SCIT, average VAS score $8.30( \pm 1.36$, SD). Patients agreed that SCIT improved their quality of life with average VAS score $8.38( \pm 1.35, S D)$. Patients' perceptions regarding SIT treatment properties before and after receiving SCIT are summarized in Table 2. Most patients satisfied with 
A

(USD)

1500

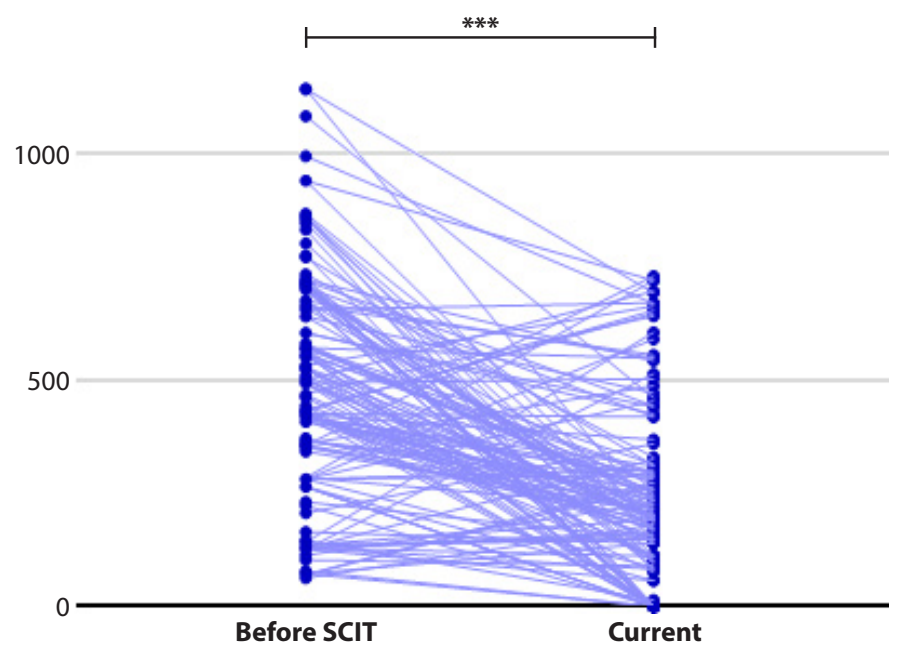

B

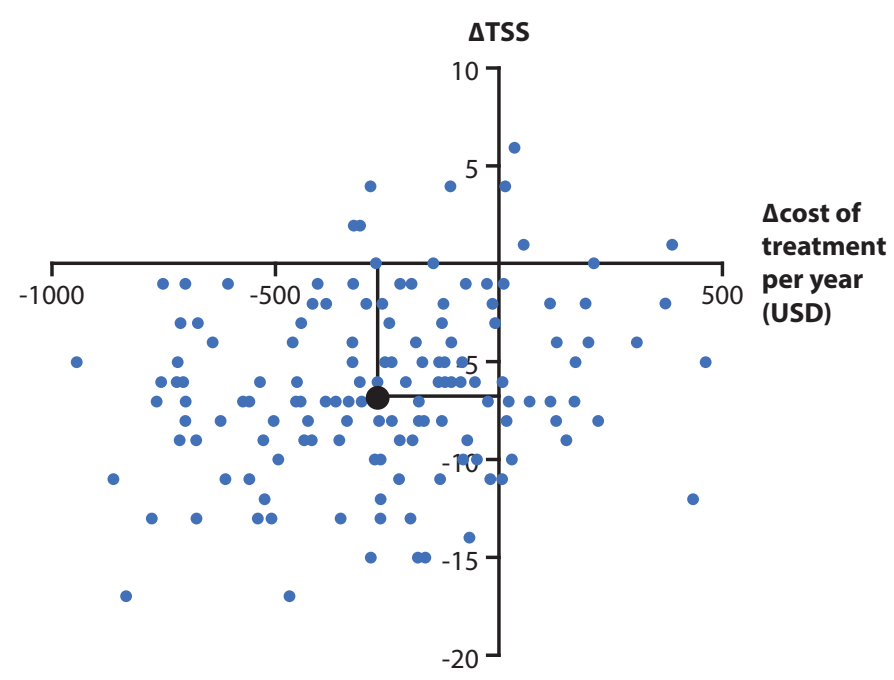

Figure 3. Direct cost-benefit of subcutaneous immunotherapy (SCIT)

A: Average current cost of medication per year including cost of SCIT was significantly lower than cost of medication before SCIT, 235.4 ( \pm 198.4 , SD) US dollars (USD) vs $489.6\left( \pm 243.3\right.$, SD) USD, ${ }^{* * * *} P<0.0001$. B: Differences of cost of medication between before receiving SCIT and current cost including cost of immunotherapy) of each patient were plotted against difference of total symptom score between before SCIT and current score. Cost of treatment decreased 254.2 USD/year by average; total symptom score (TSS) decreased 6.22 point by average.

Table 2. Patients' perception on SCIT

\begin{tabular}{|c|c|c|c|c|c|c|}
\hline \multirow[b]{2}{*}{ Questions } & & \multicolumn{4}{|c|}{ Patients' response } & \multirow[b]{2}{*}{$p$ value } \\
\hline & & $\begin{array}{l}\text { Completely } \\
\text { false (\%) }\end{array}$ & $\begin{array}{c}\text { Partly } \\
\text { false (\%) }\end{array}$ & $\begin{array}{c}\text { Partly } \\
\text { true (\%) }\end{array}$ & $\begin{array}{c}\text { Completely } \\
\text { true }(\%)\end{array}$ & \\
\hline \multirow[t]{2}{*}{ SCIT is safe } & Pre & 0.0 & 24.6 & 62.7 & 11.3 & $<0.0001$ \\
\hline & Post & 0.0 & 0.0 & 22.5 & 76.1 & \\
\hline \multirow[t]{2}{*}{ SCIT is easy to take } & Pre & 2.1 & 40.1 & 47.2 & 9.9 & $<0.0001$ \\
\hline & Post & 0.0 & 4.2 & 49.3 & 46.5 & \\
\hline \multirow[t]{2}{*}{ SCIT is handy to use in daily routine } & Pre & 0.7 & 10.6 & 65.5 & 22.5 & $<0.0001$ \\
\hline & Post & 0.0 & 0.7 & 12.0 & 87.3 & \\
\hline \multirow[t]{2}{*}{ It is easy to remember to take SCIT } & Pre & 3.5 & 25.4 & 61.3 & 9.2 & $<0.0001$ \\
\hline & Post & 0.0 & 2.1 & 31.0 & 66.2 & \\
\hline \multirow[t]{2}{*}{ SCIT allows to better control their allergy } & Pre & 0.7 & 17.6 & 64.8 & 16.2 & $<0.0001$ \\
\hline & Post & 0.0 & 0.7 & 20.4 & 78.2 & \\
\hline
\end{tabular}

Abbreviation: SCIT: subcutaneous immunotherapy

SCIT as it was a safe therapeutic option, not complicated, easy to handle and useful for their allergy treatment as the more patients agreed with each statement after receiving SCIT.

\section{Adverse reaction to SCIT}

There were 16 patients (11.3\%) experienced systemic reactions, 8 of which had reaction during rush immunotherapy:
6 grade 1 reactions (urticaria); and 2 grade 2 reactions; the patients were switched to weekly schedule at the lower concentration. During maintenance period, there were 9 systemic reactions which were 3 grade 1 reaction and 6 grade 2 reaction. Grade 2 reaction was caused by mixed HDM in 2 patients, mixed cockroach in 1 patient, mixed grass in 1 patient and mixed HDM \& mixed cockroaches in 2 patients. 


\section{Discussion}

SIT with inhalant allergen has been demonstrated to be efficacious in the treatment of IgE-mediated allergic airway diseases including AR, allergic asthma, allergic conjunctivitis..$^{14,15}$ In AR, indications for initiation of SCIT includes inadequate disease control despite of appropriate use of medication, unable to complete avoidance of allergens or patients' preferences. ${ }^{12}$ We present our data including 142 patients who were receiving SCIT or had completed maintenance phase of SCIT. Most of our patient had moderate to severe AR despite of receiving combination of medical treatment for many years.

Consistent with previous studies, ${ }^{14-17}$ both nasal and ocular symptom score of most patients decreased after receiving SCIT. In most studies, TSS decreased significantly since the first year of maintenance phase. In our study, TSS of most patients who were in the maintenance phase was decreased significantly compare to those prior SCIT.

Duration of SCIT is generally based on response to SCIT, patient's preference, and convenience of treatment. ${ }^{13}$ If SCIT is effective, discontinuation of SCIT may be considered after 3 to 5 years of treatment. However, symptoms may relapse after discontinuation, Des Roches et al reported that $55 \%$ of children with allergic asthma who received HDM SCIT for 12-96 months developed relapse of allergic symptoms within 3 years after cessation of SCIT. ${ }^{16}$ Some of contributing factors that are associated with prolonged effectiveness are longer period of maintenance phase, seasonal allergens, allergen exposure and environmental control during and after treatment. ${ }^{17,18}$ In our study, duration of SCIT in the patients who discontinued treatment ranged from 15 to 142 months. We found no correlation between duration of SCIT or number of allergens and the difference of TSS at pre and post SCIT. We found that effect of SCIT in decreasing TSS persist longer than 18 months after discontinuation.

Over a half of direct cost of AR treatment is prescription medications. ${ }^{19}$ Several studies have examined the cost-effectiveness of AIT versus pharmacotherapy alone; most studies demonstrate that SCIT is a cost-saving treatment either during treatment or after treatment. ${ }^{20,21}$ We demonstrate that SCIT was associated with $21.7 \%$ reduction of estimate direct cost of treatment in the hospital which includes medications and SCIT cost; the annual per patient cost-benefit was 215.84 USD. The significant difference in medication cost was evident within the first year after initiation of SCIT. Hence, our data of cost and effectiveness of SCIT suggest that at least three years of maintenance phase is recommended to reduce the cost of prolonged maintenance phase and indirect cost such as hospital visit or loss of income. These findings also demonstrate that SCIT can reduce the burden healthcare utilities in the hospital that payers such as the government or the National Health Security Office have to pay.

Most patients in the study experienced the improvement in quality of life after SCIT in most aspects including sleep, work/school performance and sociability. They also satisfied and had good perception in SCIT. We found very few of adverse reaction from SCIT which most of systemic reactions were due to rush immunotherapy. $11.2 \%$ of patients experienced systemic reaction. The number is quite high compare to previous reports ${ }^{22-24}$ The rate of systemic reaction from SCIT is relatively low at $0.1-0.2 \%$ with conventional build up protocols. The rate is significantly increased with accelerated build-up regimens. A half of events in our study occurred during rush immunotherapy which commonly induce systemic reaction despite of premedication. ${ }^{24}$ Skin test to allergen may be conducted again before performing rush immunotherapy as the increasing in skin test reactivity is correlated with severity of system reaction..$^{24}$

There are some limitations in our study. Firstly, the recall bias of symptom score before SCIT, it is unlikely that the patients can remember their symptoms correctly. However, we reviewed the medical records prior to SCIT to reassure that the score was correlated with the severity of disease. Secondly, we did not record rescue medication to assess the medication score which could improve the significance of our study. Lastly, complete information of both direct and indirect cost of AR treatment may demonstrate the actual cost benefit of SCIT.

In conclusion, SCIT is an effective, cost saving and safe treatment option for AR patient. Rush immunotherapy can reduce duration of build-up phase but increase the risk of systemic reaction.

\section{Acknowledgments}

We would like to thank Thammasat University for funding. We also would like to express the gratitude to all participants.

All authors declare no conflict of interest relevant to this article.

\section{References}

1. Bousquet J, Khaltaev N, Cruz AA, Denburg J, Fokkens WJ, Togias A, et al. Allergic Rhinitis and its Impact on Asthma (ARIA) 2008 update (in collaboration with the World Health Organization, GA(2)LEN and AllerGen). Allergy. 2008;63 Suppl 86:8-160.

2. Bunnag C, Jareoncharsri P, Tantilipikorn P, Vichyanond P, Pawankar R. Epidemiology and current status of allergic rhinitis and asthma in Thailand -- ARIA Asia-Pacific Workshop report. Asian Pac J Allergy Immunol. 2009;27:79-86.

3. Brozek JL, Bousquet J, Agache I, Agarwal A, Bachert C, Bosnic-Anticevich $\mathrm{S}$, et al. Allergic Rhinitis and its Impact on Asthma (ARIA) guidelines-2016 revision. J Allergy Clin Immunol. 2017;140:950-8.

4. Ahmad N, Zacharek MA. Allergic rhinitis and rhinosinusitis. Otolaryngol Clin North Am. 2008;41:267-81, v.

5. Seidman MD, Gurgel RK, Lin SY, Schwartz SR, Baroody FM, Bonner JR, et al. Clinical practice guideline: Allergic rhinitis. Otolaryngol Head Neck Surg. 2015;152:S1-43.

6. Wise SK, Lin SY, Toskala E, Orlandi RR, Akdis CA, Alt JA, et al. International Consensus Statement on Allergy and Rhinology: Allergic Rhinitis. International forum of allergy \& rhinology. 2018;8:108-352.

7. Larenas-Linnemann DES. Worldwide allergen immunotherapy guidelines: Evidence and experience-based. Allergol Immunopathol (Madr). 2017;45 Suppl 1:17-22.

8. Passalacqua G, Bagnasco D, Ferrando M, Heffler E, Puggioni F, Canonica GW. Current insights in allergen immunotherapy. Ann Allergy Asthma Immunol. 2018;120:152-4.

9. Pfaar O, Bonini S, Cardona V, Demoly P, Jakob T, Jutel M, et al. Perspectives in allergen immunotherapy: 2017 and beyond. Allergy. 2018; 73 Suppl 104:5-23.

10. Shamji MH, Durham SR. Mechanisms of allergen immunotherapy for inhaled allergens and predictive biomarkers. J Allergy Clin Immunol. 2017;140:1485-98.

11. Huang FL, Liao EC, Yu SJ. House dust mite allergy: Its innate immune response and immunotherapy. Immunobiology. 2018;223:300-2. 
12. Georgy MS, Saltoun CA. Chapter 3: Allergen immunotherapy: definition, indication, and reactions. Allergy Asthma Proc. 2012;33 Suppl 1:9-11.

13. Cox L, Nelson H, Lockey R, Calabria C, Chacko T, Finegold I, et al. Allergen immunotherapy: a practice parameter third update. J Allergy Clin Immunol. 2011;127:S1-55.

14. Dhami S, Nurmatov U, Arasi S, Khan T, Asaria M, Zaman H, et al. Allergen immunotherapy for allergic rhinoconjunctivitis: A systematic review and meta-analysis. Allergy. 2017;72:1597-631.

15. Dhami S, Kakourou A, Asamoah F, Agache I, Lau S, Jutel M, et al. Allergen immunotherapy for allergic asthma: A systematic review and meta-analysis. Allergy. 2017;72:1825-48.

16. Des Roches A, Paradis L, Knani J, Hejjaoui A, Dhivert H, Chanez P, et al. Immunotherapy with a standardized Dermatophagoides pteronyssinus extract. V. Duration of the efficacy of immunotherapy after its cessation. Allergy. 1996;51:430-3.

17. Arroabarren E, Tabar AI, Echechipia S, Cambra K, Garcia BE, Alvarez -Puebla MJ. Optimal duration of allergen immunotherapy in children with dust mite respiratory allergy. Pediatr Allergy Immunol. 2015;26:34-41.
18. Cox L, Cohn JR. Duration of allergen immunotherapy in respiratory allergy: when is enough, enough? Ann Allergy Asthma Immunol. 2007; 98:416-26.

19. Cox LS, Murphey A, Hankin C. The Cost-Effectiveness of Allergen Immunotherapy Compared with Pharmacotherapy for Treatment of Allergic Rhinitis and Asthma. Immunol Allergy Clin North Am. 2020; 40:69-85.

20. Meadows A, Kaambwa B, Novielli N, Huissoon A, Fry-Smith A, Meads C, et al. A systematic review and economic evaluation of subcutaneous and sublingual allergen immunotherapy in adults and children with seasonal allergic rhinitis. Health Technol Assess. 2013;17:vi, xi-xiv, 1-322.

21. Hankin CS, Cox L. Allergy immunotherapy: what is the evidence for cost saving? Curr Opin Allergy Clin Immunol. 2014;14:363-70.

22. Adamic K, Zidarn M, Bajrovic N, Erzen R, Kopac P, Music E. The local and systemic side-effects of venom and inhaled-allergen subcutaneous immunotherapy. Wien Klin Wochenschr. 2009;121:357-60.

23. Makatsori M, Calderon MA. Anaphylaxis: still a ghost behind allergen immunotherapy. Curr Opin Allergy Clin Immunol. 2014;14:316-22.

24. James C, Bernstein DI. Allergen immunotherapy: an updated review of safety. Curr Opin Allergy Clin Immunol. 2017;17:55-9. 